\title{
Joint Probabilistic Data Association-Feedback Particle Filter for Multiple Target Tracking Applications
}

\author{
Tao Yang, Geng Huang, Prashant G. Mehta
}

\begin{abstract}
This paper introduces a novel feedback-control based particle filter for the solution of the filtering problem with data association uncertainty. The particle filter is referred to as the joint probabilistic data association-feedback particle filter (JPDA-FPF).

The JPDA-FPF is based on the feedback particle filter introduced in our earlier papers [17], [16]. The remarkable conclusion of our paper is that the JPDA-FPF algorithm retains the innovation error-based feedback structure of the feedback particle filter, even with data association uncertainty in the general nonlinear case. The theoretical results are illustrated with the aid of two numerical example problems drawn from multiple target tracking applications.
\end{abstract}

\section{INTRODUCTION}

Filtering with data association uncertainty is important to a number of applications, including, air and missile defense systems, air traffic surveillance, weather surveillance, ground mapping, geophysical surveys, remote sensing, autonomous navigation and robotics [1], [3]. In each of these applications, there exists data association uncertainty in the sense that one can not assign individual measurements to individual targets in an apriori manner.

Given the large number of applications, algorithms for filtering problems with data association uncertainty have been extensively studied in the past; cf., [1], [10] and references therein. A typical algorithm is comprised of two parts:

(i) A filtering algorithm for tracking a single target, and

(ii) A data association algorithm for associating measurements to targets.

Prior to mid-1990s, the primary tool for filtering was a Kalman filter or one of its extensions, e.g., extended Kalman filter. The limitations of these tools in applications arise on account of nonlinearities, not only in dynamic motion of targets (e.g., drag forces in ballistic targets) but also in the measurement models (e.g., range or bearing). The nonlinearities can lead to a non-Gaussian multimodal conditional distribution. For such cases, Kalman and extended Kalman filters are known to perform poorly; cf., [15]. Since the advent and wide-spread use of particle filters [7], [6], such filters are becoming increasing relevant to single and multiple target tracking applications; cf., [15] and references therein.

Financial support from the AFOSR grant FA9550-09-1-0190 and the NSF grant EECS-0925534 is gratefully acknowledged.

T. Yang and P. G. Mehta are with the Coordinated Science Laboratory and the Department of Mechanical Science and Engineering at the University of Illinois at Urbana-Champaign (UIUC) taoyang1@illinois.edu; mehtapgeillinois.edu

G. Huang is with the Department of Electrical and Computer Engineering at UIUC huang125eillinois.edu
The other part is the data association algorithm. The purpose of the data association algorithm is to assign measurements to targets. The complications arise due to multiple non-target specific measurements (due to multiple targets in the coverage area), missing measurements (probability of detection less than one, e.g., due to target occlusion), false alarms (due to clutter) and apriori unknown number of targets (that require track initiation).

The earlier solutions considered assignments in a deterministic manner: These include the simple but non-robust "nearest neighbor" assignment algorithm and the multiple hypothesis testing (MHT) algorithm, requiring exhaustive enumeration [14], [3]. However, exhaustive enumeration leads to an NP-hard problem because number of associations increases exponentially with time.

The complexity issue led to development of probabilistic approaches: These include the probabilistic MHT or its simpler "single-scan" version, the joint probabilistic data association filter (JPDAF) [10], [2]. The central object of interest in these approaches is the computation (or approximation) of the measurement-to-target association probability. Certain modeling assumptions are necessary to compute these in a tractable fashion. Although the probabilistic algorithms have reduced computational complexity, they have primarily been developed for linear settings; cf., [1].

The development of particle filters has naturally led to investigations of data association algorithms based on importance sampling techniques. This remains an active area of research; cf., [11] and references therein. One early contribution is the multitarget particle filter (MPFT) in [8]. The data association problem is approached in the same probabilistic spirit as the basic JPDAF. The association probabilities are obtained via the use of Markov Chain Monte-Carlo (MCMC) techniques; see also [12], [13] for related approaches.

In this paper, we introduce a novel feedback control-based particle filter algorithm for solution of the joint filtering-data association problem. The proposed algorithm is based on the feedback particle filter concept introduced by us in earlier papers [17], [16]. A feedback particle filter is a controlled system to approximate the solution of the nonlinear filtering problem. The filter has a feedback structure similar to the Kalman filter: At each time $t$, the control is obtained by using a proportional gain feedback with respect to a certain modified form of the innovation error. The filter design amounts to design of the proportional gain - the solution is given by the Kalman gain in the linear Gaussian case.

In the present paper, we extend the feedback particle filter to problems with data association uncertainty. We refer 
to the resulting algorithm as the joint probabilistic data association-feedback particle filter (JPDA-FPF). As the name suggests, the proposed algorithm represents a generalization of the Kalman filter-based joint probabilistic data association (JPDAF) now to the general nonlinear filtering problem.

One remarkable conclusion of our paper is that the JPDAFPF retains the innovation error-based feedback structure even for the nonlinear problem. The innovation error-based feedback structure is expected to be useful because of the coupled nature of filtering and the data association problems.

The theoretical results are illustrated with two numerical examples. The first example considers the problem of tracking a single target in the presence of clutter. The second example considers a multiple target tracking problem. The model problem scenario is used to illustrate the performance of JPDA-FPF vis-a-vis possible track coalescence in the presence of data association uncertainty [4].

The outline of this paper is as follows: The JPDA-FPF algorithm is first described for single target in the presence of clutter, in Sec. III The multiple target case follows as a direct extension, and is discussed in Sec. IIII Numerical examples appear in Sec. IV

The theory of feedback particle filter can be found in our earlier papers [17],[16]. In the remainder of this paper, we restrict ourselves to the scalar filtering problem. The scalar case serves the pedagogical purpose, and is notationally convenient. The extension to the multivariable case is straightforward: The feedback particle filter has the same innovation error-based structure except that the gain function is replaced by the gain vector. The multivariable feedback particle filter is used in the two numerical examples described in Sec. IV

\section{Feedback Particle Filter with Data ASSOCIATION UNCERTAINTY}

In this section, we describe the probabilistic data association-feedback particle filter (PDA-FPF) for the problem of filtering a single target with multiple measurements. The filter for multiple independent targets is obtained as a straightforward extension, and briefly described in Sec. IIII

\section{A. Problem statement, Assumptions and Notation}

The following notation is adopted:

(i) At time $t$, the target state is denoted by $X_{t} \in \mathbb{R}$.

(ii) At time $t$, the observation vector $\underline{Z}_{t}:=$ $\left(Z_{t}^{1}, Z_{t}^{2}, \ldots, Z_{t}^{M}\right)^{T}$, where $M$ is assumed fixed and $Z_{t}^{m} \in \mathbb{R}$.

(iii) At time $t$, the association random variable is denoted as $A_{t} \in\{0,1, \ldots, M\}$. It is used to associate one measurement to the target: $A_{t}=m$ signifies that the $m^{\text {th }}$-measurement $Z_{t}^{m}$ is 'associated' with the target, and $A_{t}=0$ means that the target is not detected at time $t$.

The following models are assumed for the three stochastic processes:

(i) The state $X_{t}$ evolves according to a nonlinear SDE:

$$
\mathrm{d} X_{t}=a\left(X_{t}\right) \mathrm{d} t+\sigma_{B} \mathrm{~d} B_{t},
$$

where $a(\cdot)$ is a $C^{1}$ function and $\left\{B_{t}\right\}$ is a standard Wiener process.

(ii) The association random process $A_{t}$ evolves as a jump Markov process in continuous-time:

$$
\mathrm{P}\left(A_{t+\delta}=m^{\prime} \mid A_{t}=m\right)=\frac{c}{M} \delta+o(\delta), \quad m^{\prime} \neq m .
$$

The initial distribution $\mathrm{P}\left(\left[A_{0}=m\right]\right)=\frac{1}{M+1} . A_{t}$ and $X_{t}$ are assumed to be mutually independent.

(iii) At time $t$, the observation model is given by,

$$
\mathrm{d} Z_{t}^{m}=1_{\left[A_{t}=m\right]} h\left(X_{t}\right) \mathrm{d} t+\sigma_{W} \mathrm{~d} W_{t}^{m},
$$

for $m \in\{1, \ldots, M\}$, where $h(\cdot)$ is $C^{1}$ function and $\left\{W_{t}^{m}\right\}$ are mutually independent standard Wiener processes and

$$
1_{\left[A_{t}=m\right]}:= \begin{cases}1 & \text { when } A_{t}=m \\ 0 & \text { otherwise. }\end{cases}
$$

The problem is to obtain the posterior distribution of $X_{t}$ given the history of observations $\underline{\mathscr{Z}}_{t}:=\sigma\left(\underline{Z}_{s}: s \leq t\right)$.

The methodology comprises of the following two parts:

(i) Evaluation of association probability, and

(ii) Integration of association probability in the feedback particle filter framework.

\section{B. Association Probability for a Single Target}

The association probability is defined as the probability of the association $\left[A_{t}=m\right]$ conditioned on $\underline{Z}_{t}$ :

$$
\beta_{t}^{m} \triangleq \mathrm{P}\left(\left[A_{t}=m\right] \mid \underline{\mathscr{Z}}_{t}\right), \quad m=0,1, \ldots, M .
$$

Since the events are mutually exclusive and exhaustive, $\sum_{m=0}^{M} \beta_{t}^{m}=1$.

For the single-target-multiple-observation model described above, the filter for computing association probability is derived in Appendix A. It is of the following form: For $m \in\{1, \ldots, M\}$,

$$
\begin{aligned}
\mathrm{d} \beta_{t}^{m} & =\frac{c}{M}\left[1-(M+1) \beta_{t}^{m}\right] \mathrm{d} t \\
& +\frac{1}{\sigma_{W}^{2}} \beta_{t}^{m} \hat{h}_{t} \sum_{j=1}^{M} \beta_{t}^{j}\left[\left(\mathrm{~d} Z_{t}^{m}-\beta_{t}^{m} \hat{h}_{t} \mathrm{~d} t\right)-\left(\mathrm{d} Z_{t}^{j}-\beta_{t}^{j} \hat{h}_{t} \mathrm{~d} t\right)\right] \\
& +\frac{1}{\sigma_{W}^{2}} \beta_{t}^{m}\left(\widehat{h_{t}^{2}}-\hat{h}_{t}^{2}\right) \sum_{j=1}^{M} \beta_{t}^{j}\left(\beta_{t}^{j}-\beta_{t}^{m}\right) \mathrm{d} t
\end{aligned}
$$

where $\hat{h}_{t}=\mathrm{E}\left[h\left(X_{t}\right) \mid \mathscr{Z}_{t}\right]$ and $\widehat{h_{t}^{2}}=\mathrm{E}\left[h^{2}\left(X_{t}\right) \mid \mathscr{Z}_{t}\right]$. These are approximated by using the feedback particle filter described in the following section.

In practice, one may also wish to consider approaches to reduce filter complexity, e.g., by assigning gating regions for the measurements; cf., Sec. 4.2.3 in [2].

Remark 1: In the following, we integrate association probability with the feedback particle filter, which is used to approximate evolution of the posterior. Separate algorithms for data association and posterior are motivated in part by the classical JPDA filtering literature [2], [1], [10]. A separate treatment is also useful while considering multiple target tracking problems. For such problems, one can extend algorithms for data association in a straightforward manner, 
while the algorithm for posterior remains as before. This is illustrated with the aid of two-target-two-observation example in Sec III

Remark 2: The association probability filter (5) can also be derived by considering a continuous-time limit starting from the continuous-discrete time filter in literature [2]. This proof appears in Appendix D. The alternate proof is included for the following reasons:

(i) The proof shows that the filter (5) is in fact the continuous-time nonlinear counterpart of the algorithm that is used to obtain association probability in the classical JPDAF filter. This is important because some of the modeling assumptions (e.g., modeling of clutter, or of association $A_{t}$ via a jump Markov process) here may appear to be different from those considered in the classical literature.

(ii) The proof method suggests alternate discrete-time algorithms for evaluating association probabilities in simulations and experiments, where observations are made at discrete sampling times.

\section{Probabilistic Data Association-Feedback Particle Filter}

Following the feedback particle filter methodology, the model for the particle filter is given by,

$$
\mathrm{d} X_{t}^{i}=a\left(X_{t}^{i}\right) \mathrm{d} t+\sigma_{B} \mathrm{~d} B_{t}^{i}+\mathrm{d} U_{t}^{i},
$$

where $X_{t}^{i} \in \mathbb{R}$ is the state for the $i^{\text {th }}$ particle at time $t$, $U_{t}^{i}$ is its control input, and $\left\{B_{t}^{i}\right\}$ are mutually independent standard Wiener processes. We assume the initial conditions $\left\{X_{0}^{i}\right\}_{i=1}^{N}$ are i.i.d., independent of $\left\{B_{t}^{i}\right\}$, and drawn from the initial distribution $p^{*}(x, 0)$ of $X_{0}$. Both $\left\{B_{t}^{i}\right\}$ and $\left\{X_{0}^{i}\right\}$ are also assumed to be independent of $X_{t}, Z_{t}$. Certain additional assumptions are made regarding admissible forms of control input (see [16]).

Recall that there are two types of conditional distributions of interest in our analysis:

(i) $p(x, t)$ : Defines the conditional dist. of $X_{t}^{i}$ given $\underline{\mathscr{Z}}_{t}$.

(ii) $p^{*}(x, t)$ : Defines the conditional dist. of $X_{t}$ given $\underline{\mathscr{Z}}_{t}$.

The control problem is to choose the control input $U_{t}^{i}$ so that $p$ approximates $p^{*}$, and consequently empirical distribution of the particles approximates $p^{*}$ for large number of particles.

The evolution of $p^{*}(x, t)$ is described by modified form of the Kushner-Stratonovich (K-S) equation:

$$
\mathrm{d} p^{*}=\mathscr{L}^{\dagger} p^{*} \mathrm{~d} t+\frac{1}{\sigma_{W}^{2}} \sum_{m=1}^{M} \beta_{t}^{m}\left(h-\hat{h}_{t}\right)\left(\mathrm{d} Z_{t}^{m}-\hat{h}_{t} \mathrm{~d} t\right) p^{*}
$$

where $\hat{h}_{t}=\int h(x) p^{*}(x, t) \mathrm{d} x$, and $\mathscr{L}^{\dagger}$ is the Kolmogorov forward operator. The proof appears in Appendix B.

The main result of this section is to describe an explicit formula for the optimal control input, and demonstrate that under general conditions we obtain an exact match: $p=p^{*}$ under optimal control. The optimally controlled dynamics of the $i^{\text {th }}$ particle have the following form,

$$
\begin{aligned}
\mathrm{d} X_{t}^{i}=a\left(X_{t}^{i}\right) \mathrm{d} t+ & \sigma_{B} \mathrm{~d} B_{t}^{i}+\sum_{m=1}^{M} \beta_{t}^{m} \mathrm{~K}\left(X_{t}^{i}, t\right) \mathrm{d} I_{t}^{i, m} \\
& +\frac{1}{2} \sigma_{W}^{2} \sum_{m=1}^{M}\left(\beta_{t}^{m}\right)^{2} \mathrm{~K}\left(X_{t}^{i}, t\right) \mathrm{K}^{\prime}\left(X_{t}^{i}, t\right) \mathrm{d} t,
\end{aligned}
$$

where $I_{t}^{i, m}$ is a modified form of the innovation process,

$$
\mathrm{d} I_{t}^{i, m}:=\mathrm{d} Z_{t}^{m}-\left[\frac{\beta_{t}^{m}}{2} h\left(X_{t}^{i}\right)+\left(1-\frac{\beta_{t}^{m}}{2}\right) \hat{h}_{t}\right] \mathrm{d} t,
$$

where $\hat{h}_{t}:=\mathrm{E}\left[h\left(X_{t}\right) \mid \mathscr{Z}_{t}\right]=\int h(x) p(x, t) \mathrm{d} x$. The gain function $\mathrm{K}$ is the solution of a certain EL-BVP:

$$
-\frac{\partial}{\partial x}\left(\frac{1}{p(x, t)} \frac{\partial}{\partial x}\{p(x, t) \mathrm{K}(x, t)\}\right)=\frac{1}{\sigma_{W}^{2}} h^{\prime}(x),
$$

The evolution of $p(x, t)$ is easily obtained as the forward Kolmogorov operator: See Appendix $B$ for the equations.

The following theorem shows that the two evolution equations for $p$ and $p^{*}$ are identical. The proof appears in Appendix B.

Theorem 1: Consider the two evolutions for $p$ and $p^{*}$, defined according to the Kolmogorov forward equation and modified K-S equation (7), respectively. Suppose that the gain function $\mathrm{K}(X, t)$ is obtained according to (10). Then provided $p(x, 0)=p^{*}(x, 0)$, we have for all $t \leq 0, p(x, t)=$ $p^{*}(x, t)$.

Example 1: Consider the single target, single measurement case where the measurement may be due to clutter (false alarm). Let $\beta_{t}$ denote the measurement-to-target association probability at time $t$.

For this case, the feedback particle filter is given by the controlled system which is a special case of (8),

$$
\begin{aligned}
\mathrm{d} X_{t}^{i}= & a\left(X_{t}^{i}\right) \mathrm{d} t+\sigma_{B} \mathrm{~d} B_{t}^{i} \\
& +\beta_{t} \mathrm{~K}\left(X_{t}^{i}, t\right) \mathrm{d} I_{t}^{i}+\frac{1}{2} \beta_{t}^{2} \sigma_{W}^{2} \mathrm{~K}\left(X_{t}^{i}, t\right) \mathrm{K}^{\prime}\left(X_{t}^{i}, t\right) \mathrm{d} t,
\end{aligned}
$$

where the innovation error $I_{t}^{i}$ is given by,

$$
\mathrm{d} I_{t}^{i}:=\mathrm{d} Z_{t}-\left(\frac{\beta_{t}}{2} h\left(X_{t}^{i}\right)+\left(1-\frac{\beta_{t}}{2}\right) \hat{h}_{t}\right) \mathrm{d} t .
$$

For the two extreme values of $\beta_{t}$, the filter reduces to the known form:

(i) If $\beta_{t}=1$, the measurement is associated with the target with probability 1 . In this case, the filter is the same as FPF presented in [16].

(ii) If $\beta_{t}=0$, the measurement carries no information and the control input $\mathrm{d} U_{t}^{i}=0$.

For $\beta_{t} \in(0,1)$, the control is more interesting. The remarkable fact is that the innovation error-based feedback control structure is preserved. The association probability serves to modify the formulae for the gain function and the innovation error:

(i) The gain function is effectively reduced to $\beta_{t} \mathrm{~K}\left(X_{t}^{i}, t\right)$. That is, the control gets less agressive in the presence of possible false alarms due to clutter. 
(ii) The innovation error is given by a more general formula (12). The optimal prediction of the $i^{\text {th }}$-particle is now a weighted average of $h\left(X_{t}^{i}\right)$ and the population prediction $\hat{h}_{t} \approx \frac{1}{N} \sum_{j=1}^{N} h\left(X_{t}^{j}\right)$. Effectively, in the presence of possible false alarms, a particle gives more weight to the population in computing its innovation error.

\section{Example: Linear Case}

We provide here a special case of PDA-FPF for the single target tracking problem described by a linear model:

$$
\begin{aligned}
& \mathrm{d} X_{t}=\alpha X_{t} \mathrm{~d} t+\sigma_{B} \mathrm{~d} B_{t}, \\
& \mathrm{~d} Z_{t}=\gamma X_{t} \mathrm{~d} t+\sigma_{W} \mathrm{~d} W_{t},
\end{aligned}
$$

where $\alpha, \gamma$ are real numbers.

The PDA-FPF is described by (8)-10). If we assume $p(x, t)$ to be Gaussian at each time with mean $\mu_{t}$ and variance $\Sigma_{t}$, i.e., $p(x, t)=\frac{1}{\sqrt{2 \pi \Sigma_{t}}} \exp \left(-\frac{\left(x-\mu_{t}\right)^{2}}{2 \Sigma_{t}}\right)$, then by direct substitution in (10) we obtain the gain function:

$$
\mathrm{K}(x, t)=\frac{\gamma \Sigma_{t}}{\sigma_{W}^{2}}
$$

The PDA-FPF is then given by,

$$
\begin{aligned}
\mathrm{d} X_{t}^{i} & =\alpha X_{t}^{i} \mathrm{~d} t+\sigma_{B} \mathrm{~d} B_{t}^{i} \\
& +\frac{\gamma \Sigma_{t}}{\sigma_{W}^{2}} \sum_{m=1}^{M} \beta_{t}^{m}\left[\mathrm{~d} Z_{t}^{m}-\gamma\left(\frac{\beta_{t}^{m}}{2} X_{t}^{i}+\left(1-\frac{\beta_{t}^{m}}{2}\right) \mu_{t}\right) \mathrm{d} t\right] .
\end{aligned}
$$

The filter for association probability $\beta_{t}^{m}$ is as follows:

$$
\begin{aligned}
& \mathrm{d} \beta_{t}^{m}=\frac{c}{M}\left[1-(M+1) \beta_{t}^{m}\right] \mathrm{d} t \\
& +\frac{1}{\sigma_{W}^{2}} \beta_{t}^{m} \gamma \mu_{t} \sum_{j=1}^{M} \beta_{t}^{j}\left[\left(\mathrm{~d} Z_{t}^{m}-\beta_{t}^{m} \gamma \mu_{t} \mathrm{~d} t\right)-\left(\mathrm{d} Z_{t}^{j}-\beta_{t}^{j} \gamma \mu_{t} \mathrm{~d} t\right)\right] \\
& \quad+\frac{1}{\sigma_{W}^{2}} \beta_{t}^{m} \gamma^{2} \Sigma_{t} \sum_{j=1}^{M} \beta_{t}^{j}\left(\beta_{t}^{j}-\beta_{t}^{m}\right) \mathrm{d} t
\end{aligned}
$$

In practice $\left\{\mu_{t}, \Sigma_{t}\right\}$ in (15)-(16) are approximated as sample means and sample covariances using $\left\{X_{t}^{i}\right\}_{i=1}^{N}$.

$$
\begin{aligned}
& \mu_{t} \approx \mu_{t}^{(N)}:=\frac{1}{N} \sum_{i=1}^{N} X_{t}^{i} \\
& \Sigma_{t} \approx \Sigma_{t}^{(N)}:=\frac{1}{N-1} \sum_{i=1}^{N}\left(X_{t}^{i}-\mu_{t}^{(N)}\right)^{2} .
\end{aligned}
$$

\section{Multiple target Tracking using FEEDBACK PARTICLE FiLTER}

In this section, we extend the PDA-FPF to multiple target tracking problems. Specifically, a two-target two-observation problem is used to illustrate JPDA-FPF. The extension to the more general case is straightforward.

\section{A. Problem statement}

The following notation is adopted:

(i) At time $t$, the target state is denoted as $\underline{X}_{t}:=\left(X_{t}^{1}, X_{t}^{2}\right)^{T}$, where $X_{t}^{n} \in \mathbb{R}$ for $n \in\{1,2\}$.

(ii) At time $t$, the observation vector $\underline{Z}_{t}:=\left(Z_{t}^{1}, Z_{t}^{2}\right)^{T}$, where $Z_{t}^{m} \in \mathbb{R}$ for $m \in\{1,2\}$.

(iii) At time $t$, the association random variable is denoted as $A_{t} \in\{1,2\}$. It is used to associate measurements to targets in a joint manner: $A_{t}=1$ signifies that $Z_{t}^{1}$ is associated with target 1 and $Z_{t}^{2}$ with target 2 . Similarly $A_{t}=2$ accounts for the complementary case.

The following models are assumed for the three stochastic processes:

(i) Each element of the state vector $\underline{X}_{t}$ evolves according to a one-dimensional nonlinear SDE:

$$
\mathrm{d} X_{t}^{n}=a\left(X_{t}^{n}\right) \mathrm{d} t+\sigma_{B}^{n} \mathrm{~d} B_{t}^{n}, \quad n \in\{1,2\}
$$

where $\left\{B_{t}^{1}\right\},\left\{B_{t}^{2}\right\}$ are mutually independent standard Wiener processes.

(ii) The association random process $A_{t}$ evolves as a jump Markov process in continuous-time:

$$
\mathrm{P}\left(A_{t+\delta}=m^{\prime} \mid A_{t}=m\right)=c \delta+o(\delta), \quad m^{\prime} \neq m .
$$

The initial distribution $\mathrm{P}\left(\left[A_{0}=m\right]\right)=\frac{1}{2} . A_{t}$ and $\underline{X}_{t}$ are assumed to be mutually independent.

(iii) At time $t$, the observation model is given by,

$$
\left[\begin{array}{c}
\mathrm{d} Z_{t}^{1} \\
\mathrm{~d} Z_{t}^{2}
\end{array}\right]=\Psi\left(A_{t}\right)\left[\begin{array}{c}
h\left(X_{t}^{1}\right) \\
h\left(X_{t}^{2}\right)
\end{array}\right] \mathrm{d} t+\sigma_{W}\left[\begin{array}{c}
\mathrm{d} W_{t}^{1} \\
\mathrm{~d} W_{t}^{2}
\end{array}\right],
$$

where $\left\{W_{t}^{1}\right\},\left\{W_{t}^{2}\right\}$ are mutually independent standard Wiener processes and $\Psi\left(A_{t}\right)$ is a function which maps $A_{t}$ to a permutation matrix:

$$
\Psi(1)=\left[\begin{array}{ll}
1 & 0 \\
0 & 1
\end{array}\right], \quad \Psi(2)=\left[\begin{array}{ll}
0 & 1 \\
1 & 0
\end{array}\right] .
$$

\section{B. Joint Probabilistic Data Association for Two Target}

The joint association probability is defined as the probability of the joint association $\left[A_{t}=m\right]$ conditioned on $\underline{Z}_{t}$ :

$$
\pi_{t}^{m} \triangleq \mathrm{P}\left(\left[A_{t}=m\right] \mid \underline{\mathscr{Z}}_{t}\right), \quad m=1,2 .
$$

The filter for joint association probability $\pi_{t}$ is a straightforward extension of (5). The proof appears in Appendix $\mathrm{C}$ It is of the following form:

$$
\begin{gathered}
\mathrm{d} \pi_{t}^{1}=-c\left(\pi_{t}^{1}-\pi_{t}^{2}\right) \mathrm{d} t+\frac{1}{\sigma_{W}^{2}} \pi_{t}^{1} \pi_{t}^{2}\left(\hat{h}_{t}^{1}-\hat{h}_{t}^{2}\right)\left(\mathrm{d} \mu_{t}^{1}-\mathrm{d} \mu_{t}^{2}\right) \\
-\frac{1}{\sigma_{W}^{2}} \pi_{t}^{1} \pi_{t}^{2}\left(\pi_{t}^{1}-\pi_{t}^{2}\right)\left[\widehat{\left(h_{t}^{1}\right)^{2}}-\left(\hat{h}_{t}^{1}\right)^{2}+\widehat{\left(h_{t}^{2}\right)^{2}}-\left(\left(\hat{h}_{t}^{2}\right)^{2}\right] \mathrm{d} t\right.
\end{gathered}
$$

where $\mathrm{d} \mu_{t}^{m}=\mathrm{d} Z_{t}^{m}-\left(\pi_{t}^{1}-\pi_{t}^{2}\right) \hat{h}_{t}^{m} \mathrm{~d} t, \hat{h}_{t}^{n}:=\mathrm{E}\left[h\left(X_{t}^{n}\right) \mid \underline{\mathscr{Z}}_{t}\right]$ and $\widehat{\left(h_{t}^{n}\right)^{2}}:=\mathrm{E}\left[h^{2}\left(X_{t}^{n}\right) \mid \underline{Z}_{t}\right]$. Since the joint events are mutually exclusive and exhaustive, we have $\sum_{m=1}^{2} \pi_{t}^{m}=1$. Using this, we have $\pi_{t}^{2}=1-\pi_{t}^{1}$ and $\mathrm{d} \pi_{t}^{2}=-\mathrm{d} \pi_{t}^{1}$. 


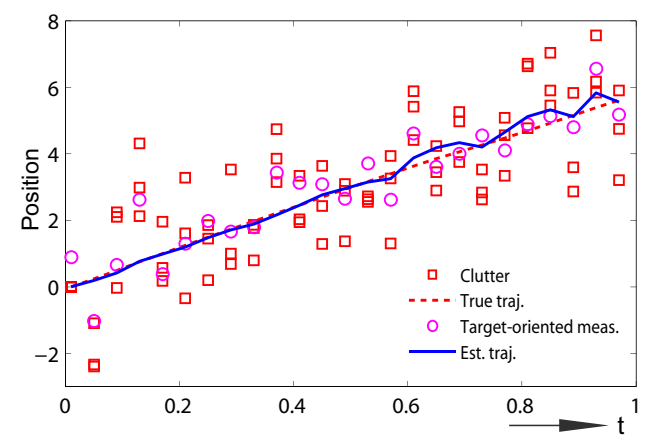

Fig. 1. Simulation results of single target tracking in clutter using PDAFPF: Comparison of estimated mean with the true trajectory.

\section{Joint Prob. Data Association-Feedback Particle Filter}

The joint association probabilities $\pi_{t}^{1}, \pi_{t}^{2}$ are used to obtain marginal association probability for individual target. For example, for target 1: $\beta_{t}^{1}=\pi_{t}^{1}, \beta_{t}^{2}=\pi_{t}^{2}$. Once the association probabilities are known, the feedback particle filter for each target is of the form (8).

\section{NUMERICS}

In this section, we discuss results of two numerical examples. Even though the theory was described for real-valued state and observation processes, the numerical examples consider more realistic multivariable models.

\section{A. Single Target Tracking in Clutter}

We first consider a single target tracking problem where the target dynamics evolve according to a white-noise acceleration model:

$$
\begin{aligned}
& \mathrm{d} X_{t}=F X_{t} \mathrm{~d} t+\sigma_{B} \mathrm{~d} B_{t}, \\
& \mathrm{~d} Z_{t}=H X_{t} \mathrm{~d} t+\sigma_{W} \mathrm{~d} W_{t},
\end{aligned}
$$

where $X_{t}$ denotes the state vector comprising of position and velocity coordinates at time $t, Z_{t}$ is the observation process, $\left\{B_{t}\right\},\left\{W_{t}\right\}$ are mutually independent standard Wiener processes. The two matrices are given by:

$$
F=\left[\begin{array}{ll}
0 & 1 \\
0 & 0
\end{array}\right], \quad H=\left[\begin{array}{ll}
1 & 0
\end{array}\right]
$$

In the simulation results described next, we use the following parameter values: $\sigma_{B}=[0 ; 1], \sigma_{W}=0.06$ and initial condition $X_{0}=[0 ; 6]$. The total simulation time is $T=1$ and time step $\mathrm{d} t=0.01$. At each discrete-time step, we assume $M=4$ measurements, one due to target and other three due to clutter. The associations are not apriori known.

Figure 1 depicts the result of a single simulation: True target trajectory is depicted as a dashed line. At each discrete time step, target-oriented measurements are depicted as circles while clutter measurements are depicted as squares. The estimated mean trajectory is depicted as a solid line. It is obtained using the PDA-FPF algorithm described in Sec IID. For the filter simulation, we use $N=1000$ particles.

\section{B. Track Coalescence Avoidance using JPDA-FPF}

Track coalescence is a common problem in multiple tracking applications. Track coalescence can occur when two closely spaced targets move with approximately the same velocity over a time period [3]. With standard implementations of JPDAF and SIR particle filter algorithms, the target tracks tends to coalesce even after the targets have moved apart [4]. In the following example, we describe simulation results for JPDA-FPF for a model problem scenario taken from [5].

We consider two targets. For each target, the dynamics are described by a white-noise acceleration model as in the preceding example. For $n=1,2$ :

$$
\mathrm{d} X_{t}^{n}=F X_{t}^{n} \mathrm{~d} t+\sigma_{B} \mathrm{~d} B_{t},
$$

where the state $X_{t}^{n}$ comprises of target position and velocity.

We assume two observations are given by

$$
\left[\begin{array}{l}
\mathrm{d} Z_{t}^{1} \\
\mathrm{~d} Z_{t}^{2}
\end{array}\right]=\Psi\left(A_{t}\right)\left[\begin{array}{c}
H X_{t}^{1} \\
H X_{t}^{2}
\end{array}\right] \mathrm{d} t+\sigma_{W}\left[\begin{array}{c}
\mathrm{d} W_{t}^{1} \\
\mathrm{~d} W_{t}^{2}
\end{array}\right],
$$

where $A_{t}$ is the association random variable, $\Psi\left(A_{t}\right)$ is the permutation matrix as defined in (21). $\left\{B_{t}\right\},\left\{W_{t}^{1}\right\},\left\{W_{t}^{2}\right\}$ are mutually independent standard Winer processes.

In the simulation results described next, we use the following parameter values: $\sigma_{B}=[0 ; 2], \sigma_{W}=[0.005 ; 0.005]$ and initial condition $\underline{X}_{0}=[1 ;-3.5 ;-1 ; 3.5]$. The total simulation time is $T=1 \mathrm{~s}$ and time step $\mathrm{d} t=0.001 \mathrm{~s}$. The prior association probability $\left(\pi_{t}^{1}, \pi_{t}^{2}\right)$ is assumed to be $(1 / 2,1 / 2)$.

Figure 2(a) depicts the results of a single simulation: The estimated mean trajectories are obtained using the JPDAFPF described in Sec III Figure 2(b) depicts the evolution of association probability $\left(\pi_{t}^{1}, \pi_{t}^{2}\right)$ during the same simulation run. For the filter simulation, we use $N=1000$ particles. To obtain the association probabilities, we use an adaptive time stepping scheme for numerical integration of association probability filter (23).

\section{ACKNOWLEDGEMENT}

We are grateful to Prof. Sean Meyn for useful discussions on the work described in this paper.

\section{APPENDIX}

\section{A. Association Probability Filter for $\beta_{t}^{m}$}

Rewrite (3) in the vector form:

$$
\mathrm{d} \underline{Z}_{t}=\underline{\chi}\left(A_{t}\right) h\left(X_{t}\right) \mathrm{d} t+\sigma_{W} \mathrm{~d} \underline{W}_{t},
$$

where $\underline{\chi}\left(A_{t}\right):=\left(\chi_{t}^{1}, \ldots, \chi_{t}^{M}\right)^{T}, \chi_{t}^{m}=\mathbf{1}_{\left[A_{t}=m\right]}$, and $\underline{W}_{t}:=$ $\left(W_{t}^{1}, \ldots, W_{t}^{M}\right)^{T}$. The transition intensity matrix for the jump Markov process $A_{t}$ is denoted as $\Lambda$ with

$$
\Lambda_{m m^{\prime}}= \begin{cases}-c & \text { if } m=m^{\prime} \\ c / M & \text { if } m \neq m^{\prime} .\end{cases}
$$

Denote $\mathscr{X}_{t}:=\sigma\left(X_{s}: s \leq t\right)$, and $\mathscr{C}_{t}:=\mathscr{X}_{t} \vee \mathscr{Z}_{t}$. The derivation is based on the property of the conditional expectation:

$$
\mathrm{E}\left[\underline{\varphi}\left(A_{t}\right) \mid \mathscr{Z}_{t}\right]=\mathrm{E}\left[\mathrm{E}\left[\underline{\varphi}\left(A_{t}\right) \mid \mathscr{C}_{t}\right] \mid \mathscr{Z}_{t}\right] .
$$



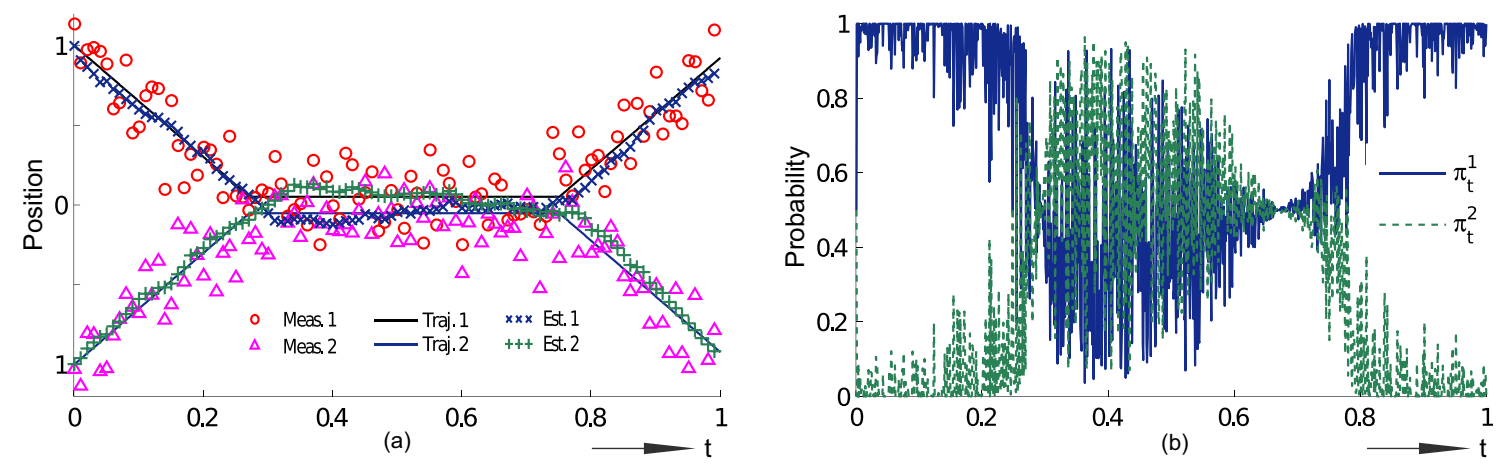

Fig. 2. Track coalescence avoiding using JPDA-FPF: (a) Comparison of estimated mean JPDA-FPF with true trajectories. (b) Plot of data association probability.

The SDE for evolution of $\mathrm{E}\left[\underline{\varphi}\left(A_{t}\right) \mid \mathscr{C}_{t}\right]$ is described by the standard Wonham filter:

$$
\begin{aligned}
& \mathrm{E}\left[\underline{\varphi}\left(A_{t}\right) \mid \mathscr{C}_{t}\right]=\mathrm{E}\left[\underline{\varphi}\left(A_{0}\right)\right]+\int_{0}^{t} \mathrm{E}\left[\Lambda \underline{\varphi}\left(A_{s}\right) \mid \mathscr{C}_{s}\right] \mathrm{d} s \\
& +\sum_{m=1}^{M} \int_{0}^{t} \mathrm{E}\left[\left(D_{s}^{m}-h\left(X_{s}\right) \beta_{s}^{m} I\right) \underline{\varphi}\left(A_{s}\right) \mid \mathscr{C}_{s}\right]\left(\mathrm{d} Z_{s}^{m}-h\left(X_{s}\right) \beta_{s}^{m} \mathrm{~d} s\right),
\end{aligned}
$$

where $I$ is the identity matrix, $D_{t}^{m}$ is the diagonal matrix where the only non-zero entry is $\left(D_{t}^{m}\right)_{m m}=h\left(X_{t}\right)$.

Taking $\mathrm{E}\left[\cdot \mid \mathscr{Z}_{t}\right]$ of (31) gives the desired result (5).

\section{B. Consistency proof of $p$ and $p^{*}$}

Evolution equation for $p^{*}$ : Recall $\mathscr{Z}_{t}:=\sigma\left(\underline{Z}_{s}: s \leq t\right), \mathscr{A}_{t}:=$ $\sigma\left(A_{s}: s \leq t\right)$. We denote $\mathscr{C}_{t}:=\mathscr{A}_{t} \vee \mathscr{Z}_{t}$. The derivation is based on the property of the conditional expectation:

$$
\mathrm{E}\left[\varphi\left(X_{t}\right) \mid \underline{\mathscr{Z}}_{t}\right]=\mathrm{E}\left[\mathrm{E}\left[\varphi\left(X_{t}\right) \mid \mathscr{C}_{t}\right] \mid \underline{\mathscr{Z}}_{t}\right] .
$$

The sde for evolution of $\mathrm{E}\left[\varphi\left(X_{t}\right) \mid \mathscr{C}_{t}\right]$ is described by the standard nonlinear filter with innovation error, $\sum_{m=1}^{M} \chi_{t}^{m}\left(\mathrm{~d} Z_{t}^{m}-\right.$ $\left.\hat{h}_{t} \mathrm{~d} t\right)$, where $\chi_{t}^{m}=\mathbf{1}_{\left[A_{t}=m\right]}$ :

$$
\begin{aligned}
\mathrm{E}\left[\varphi\left(X_{t}\right) \mid \mathscr{C}_{t}\right] & =\mathrm{E}\left[\varphi\left(X_{0}\right)\right]+\int_{0}^{t} \mathrm{E}\left[\mathscr{L} \varphi\left(X_{s}\right) \mid \mathscr{C}_{s}\right] \mathrm{d} s \\
& +\sum_{m=1}^{M} \int_{0}^{t} \mathrm{E}\left[\left(h-\hat{h}_{s}\right) \varphi\left(X_{s}\right) \mid \mathscr{C}_{s}\right] \chi_{s}^{m}\left(\mathrm{~d} Z_{s}^{m}-\hat{h}_{s} \mathrm{~d} s\right),
\end{aligned}
$$

where $\mathscr{L}$ denotes the Kolmogorov's backward operator for the diffusion (1) (the adjoint of $\mathscr{L}^{\dagger}$ ).

Taking $\mathrm{E}\left[\cdot \mid \mathscr{Z}_{t}\right]$ gives the desired result because $\mathrm{E}\left[\chi_{s}^{m} \mid \underline{\mathscr{Z}}_{s}\right]=\mathrm{P}\left(\left[A_{s}=m\right] \mid \underline{\mathscr{Z}}_{s}\right)=\beta_{s}^{m}$.

Evolution equation for $p$ : We express the FPF $(8)$ as:

$$
\mathrm{d} X_{t}^{i}=a\left(X_{t}^{i}\right) \mathrm{d} t+\sigma_{B} \mathrm{~d} B_{t}^{i}+\mathrm{K}\left(X_{t}^{i}, t\right) \sum_{m=1}^{M} \beta_{t}^{m} \mathrm{~d} Z_{t}^{m}+u\left(X_{t}^{i}, t\right) \mathrm{d} t,
$$

where

$$
\begin{aligned}
u(x, t):= & -\sum_{m=1}^{M} \beta_{t}^{m}\left[\frac{\beta_{t}^{m}}{2} h+\left(1-\frac{\beta_{t}^{m}}{2}\right) \hat{h}_{t}\right] \mathrm{K}(x, t) \\
& +\frac{\sigma_{W}^{2}}{2} \sum_{j=1}^{M}\left(\beta_{t}^{m}\right)^{2} \mathrm{KK}^{\prime}(x, t) .
\end{aligned}
$$

The evolution equation for $p$ now follows:

$$
\begin{aligned}
\mathrm{d} p=\mathscr{L}^{\dagger} p \mathrm{~d} t & -\frac{\partial}{\partial x}(u p) \mathrm{d} t+\frac{\sigma_{W}^{2}}{2} \sum_{j=1}^{M}\left(\beta_{t}^{m}\right)^{2} \frac{\partial^{2}}{\partial x^{2}}\left(p \mathrm{~K}^{2}\right) \mathrm{d} t \\
& -\frac{\partial}{\partial x}(\mathrm{~K} p) \sum_{m=1}^{M} \beta_{t}^{m} \mathrm{~d} Z_{t}^{m} .
\end{aligned}
$$

Proof of consistency. The proof follows closely the consistency proof for the feedback particle filter (see Appendix C in [16]). If $\mathrm{K}$ solves the E-L BVP then

$$
-\frac{\partial}{\partial x}(\mathrm{~K} p)=\frac{1}{\sigma_{W}^{2}}\left(h-\hat{h}_{t}\right) p
$$

On multiplying both sides of (32) by $-p$ and simplifying (by using (34)), we obtain

$$
-u p=-\frac{\sigma_{W}^{2}}{2} \frac{\partial}{\partial x}\left(p \mathrm{~K}^{2}\right) \sum_{m=1}^{M}\left(\beta_{t}^{m}\right)^{2}+\mathrm{K} p \sum_{m=1}^{M} \beta_{t}^{m} \hat{h}_{t}
$$

Differentiate now both sides with respect to $x$ and use (34) once again to arrive at

$\frac{\sigma_{W}^{2}}{2} \sum_{j=1}^{M}\left(\beta_{t}^{m}\right)^{2} \frac{\partial^{2}}{\partial x^{2}}\left(p \mathrm{~K}^{2}\right)-\frac{\partial}{\partial x}(u p)=\frac{-1}{\sigma_{W}^{2}}\left(h-\hat{h}_{t}\right) p \sum_{m=1}^{M} \beta_{t}^{m} \hat{h}_{t}$.

Using (34) and (35) in the forward equation (33), we obtain:

$$
\mathrm{d} p=\mathscr{L}^{\dagger} p \mathrm{~d} t+\frac{1}{\sigma_{W}^{2}} \sum_{j=1}^{M} \beta_{t}^{m}\left(h-\hat{h}_{t}\right)\left(\mathrm{d} Z_{t}^{m}-\hat{h}_{t} \mathrm{~d} t\right) p
$$

This is precisely the SDE (7), as desired.

\section{Association Probability filter for $\pi_{t}^{m}$}

The derivation follows closely the derivation in Appendix A Note that the observation model is described by (20). Denote $\underline{h}\left(\underline{X}_{t}\right):=\left(h\left(X_{t}^{1}\right), h\left(X_{t}^{2}\right)\right)^{T}, \widehat{\Psi}_{t}:=$ $\sum_{m=1}^{2} \pi_{t}^{m} \Psi(m)$ and $\underline{\phi}_{t}=\left(\phi_{t}^{1}, \phi_{t}^{2}\right)^{T}:=\widehat{\Psi}_{t} \underline{h}\left(\underline{X}_{t}\right)$. The Wonham filter is given by:

$$
\begin{aligned}
& \mathrm{E}\left[\underline{\varphi}\left(A_{t}\right) \mid \mathscr{C}_{t}\right]=\mathrm{E}\left[\underline{\varphi}\left(A_{0}\right)\right]+\int_{0}^{t} \mathrm{E}\left[\Lambda \underline{\varphi}\left(A_{s}\right) \mid \mathscr{C}_{s}\right] \mathrm{d} s \\
& +\sum_{m=1}^{2} \int_{0}^{t} \mathrm{E}\left[\left(D_{s}^{m}-\phi_{s}^{m} I\right) \underline{\varphi}\left(A_{s}\right) \mid \mathscr{C}_{s}\right]\left(\mathrm{d} Z_{s}^{m}-\phi_{s}^{m} \mathrm{~d} s\right),
\end{aligned}
$$


where $D_{t}^{m}$ is a $2 \times 2$ diagonal matrix where $\left(D_{t}^{m}\right)_{i i}$ is the $m^{\text {th }}$ entry of the vector $\Psi(i) \underline{h}\left(\underline{X}_{t}\right)$.

Taking $\mathrm{E}\left[\cdot \mid \mathscr{Z}_{t}\right]$ of $(37)$ gives the desired result.

\section{Alternate Derivation of (5)}

The aim of this section is to derive, formally, the update part of the continuous time filter (5) by taking a continuous time limit of the discrete-time algorithm for evaluation of association probability. The procedure for taking the limit is similar to Sec 6.8 in [9] for derivation of the K-S equation.

At time $t$, we have $M$ measurements $\mathrm{d} \underline{Z}_{t}=$ $\left(\mathrm{d} Z_{t}^{1}, \mathrm{~d} Z_{t}^{2}, \ldots, \mathrm{d} Z_{t}^{M}\right)^{T}$, only one of which originates from the target. The discrete-time filter for association probability is obtained by using Bayes' rule (see [2]):

$\mathrm{P}\left(\left[A_{t}=m\right] \mid \underline{\mathscr{Z}}_{t}, \mathrm{~d} \underline{Z}_{t}\right)=\frac{\mathrm{P}\left(\mathrm{d} \underline{Z}_{t} \mid\left[A_{t}=m\right]\right) \mathrm{P}\left(\left[A_{t}=m\right] \mid \mathscr{Z}_{t}\right)}{\sum_{j=1}^{M} \mathrm{P}\left(\mathrm{d} \underline{Z}_{t} \mid\left[A_{t}=j\right]\right) \mathrm{P}\left(\left[A_{t}=j\right] \mid \underline{\mathscr{Z}}_{t}\right)}$.

In evaluation of the association probability, one typically assumes a clutter model whereby the independent measurements are uniformly and independently distributed in the coverage area $V$ ([2],[1]). We then have:

$$
\begin{aligned}
\mathrm{P}\left(\mathrm{d} \underline{Z}_{t} \mid\left[A_{t}=m\right]\right) & =V^{1-M} \mathrm{P}\left(\mathrm{d} Z_{t}^{m} \mid\left[A_{t}=m\right]\right) \\
& =V^{1-M} L\left(\mathrm{~d} Z_{t}^{m}\right) .
\end{aligned}
$$

where $L\left(\mathrm{~d} Z_{t}^{m}\right)=\frac{1}{\sqrt{2 \pi \sigma_{W}^{2} \mathrm{~d} t}} \int_{\mathbb{R}} \exp \left[-\frac{\left(\mathrm{d} Z_{t}^{m}-h(x) \mathrm{d} t\right)^{2}}{2 \sigma_{W}^{2} \mathrm{~d} t}\right] p(x, t) \mathrm{d} x$.

Now, denote $\beta_{t}^{m}=\mathrm{P}\left(\left[A_{t}=m\right] \mid \mathscr{Z}_{t}\right)$, the increment in the measurement update step (see Sec 6.8 in [9]) is given by

$$
\mathrm{d} \beta_{t}^{m}:=\mathrm{P}\left(\left[A_{t}=m\right] \mid \underline{\mathscr{Z}}_{t}, \mathrm{~d} Z_{t}\right)-\mathrm{P}\left(\left[A_{t}=m\right] \mid \underline{\mathscr{Z}}_{t}\right) .
$$

Using (38) and (40), we have:

$$
\mathrm{d} \beta_{t}^{m}=E^{m}\left(\mathrm{~d} t, \mathrm{~d} \underline{Z}_{t}\right) \beta_{t}^{m}-\beta_{t}^{m},
$$

where

$$
E^{m}\left(\mathrm{~d} t, \mathrm{~d} \underline{Z}_{t}\right)=\frac{\mathrm{P}\left(\left[A_{t}=m\right] \mid \mathscr{Z}_{t}, \mathrm{~d} \underline{Z}_{t}\right)}{\mathrm{P}\left(\left[A_{t}=m\right] \mid \underline{\mathscr{Z}}_{t}\right)} .
$$

We expand $E^{m}\left(\mathrm{~d} t, \mathrm{~d} \underline{Z}_{t}\right)$ as a multivariate series about $(0, \underline{0})$ :

$$
\begin{aligned}
E^{m}\left(\mathrm{~d} t, \mathrm{~d} \underline{Z}_{t}\right) & =E^{m}(0, \underline{0})+E_{\mathrm{d} t}^{m}(0, \underline{0}) \mathrm{d} t+\sum_{j=1}^{M} E_{\mathrm{d} Z_{t}^{j}}^{m}(0, \underline{0}) \mathrm{d} Z_{t}^{j} \\
& +\frac{1}{2} \sum_{j, k=1}^{M} E_{\mathrm{d} Z_{t}^{j}, \mathrm{~d} Z_{t}^{k}}^{m}(0, \underline{0}) \mathrm{d} Z_{t}^{j} \mathrm{~d} Z_{t}^{k}+o(\mathrm{~d} t) .
\end{aligned}
$$

By direct evaluation, we obtain:

$$
\begin{aligned}
& E^{m}(0, \underline{0})=1, E_{\mathrm{d} t}^{m}(0, \underline{0})=0, \\
& E_{\mathrm{d} Z_{t}^{j}}^{m}(0, \underline{0})=-\frac{1}{\sigma_{W}^{2}} \beta_{t}^{j} \hat{h}_{t}, j \neq m \\
& E_{\mathrm{d} Z_{t}^{m}}^{m}(0, \underline{0})=\frac{1}{\sigma_{W}^{2}}\left(1-\beta_{t}^{m}\right) \hat{h}_{t}, \\
& E_{\mathrm{d} Z_{t}^{j}, \mathrm{~d} Z_{t}^{j}}^{m}(0, \underline{0})=\frac{1}{\sigma_{W}^{4}} \beta_{t}^{j}\left(2 \beta_{t}^{j}-1\right) \widehat{h_{t}^{2}}, j \neq m \\
& E_{\mathrm{d} Z_{t}^{m}, \mathrm{~d} Z_{t}^{m}}^{m}(0, \underline{0})=\frac{1}{\sigma_{W}^{4}}\left(1-\beta_{t}^{m}\right)\left(1-2 \beta_{t}^{m}\right) \widehat{h_{t}^{2}},
\end{aligned}
$$

where $\hat{h}_{t}:=\mathrm{E}\left[h\left(X_{t}\right) \mid \underline{\mathscr{Z}}_{t}\right]$ and $\widehat{h_{t}^{2}}:=\mathrm{E}\left[h^{2}\left(X_{t}\right) \mid \underline{\mathscr{Z}}_{t}\right]$.
By using Itô's rules,

$$
\mathrm{d} Z_{t}^{j} \mathrm{~d} Z_{t}^{k}= \begin{cases}\sigma_{W}^{2} \mathrm{~d} t, & \text { if } \mathrm{j}=\mathrm{k}, \\ 0, & \text { otherwise. }\end{cases}
$$

This gives

$$
\begin{aligned}
E^{m}\left(\mathrm{~d} t, \mathrm{~d} \underline{Z}_{t}\right)= & 1+\frac{1}{\sigma_{W}^{2}} \hat{h}_{t} \sum_{j=1}^{M} \beta_{t}^{j}\left(\mathrm{~d} Z_{t}^{m}-\mathrm{d} Z_{t}^{j}\right) \\
& +\frac{1}{\sigma_{W}^{2}} \widehat{h_{t}^{2}} \sum_{j=1}^{M} \beta_{t}^{j}\left(\beta_{t}^{j}-\beta_{t}^{m}\right) \mathrm{d} t,
\end{aligned}
$$

Substituting (44) to (41) we otain the expression for $\mathrm{d} \beta_{t}^{m}$ which equals the measurement update part of the continuoustime filter (5).

Remark 3: During a discrete-time implementation, one can use (38)-(39) to obtain association probability. In (38), $L\left(\mathrm{~d} Z_{t}^{m}\right)$ is approximated by using particles:

$$
\begin{gathered}
L\left(\mathrm{~d} Z_{t}^{m}\right) \approx \frac{1}{N} \frac{1}{\sqrt{2 \pi \sigma_{W}^{2} \mathrm{~d} t}} \sum_{i=1}^{N} \exp \left[-\frac{\left(\mathrm{d} Z_{t}^{m}-h\left(X_{t}^{i}\right) \mathrm{d} t\right)^{2}}{2 \sigma_{W}^{2} \mathrm{~d} t}\right] . \\
\text { REFERENCES }
\end{gathered}
$$

\section{REFERENCES}

[1] Y. Bar-Shalom, F. Daum, and J. Huang. The probabilistic data association filter. IEEE Control Systems Magazine, 29(6):82-100, Dec 2009.

[2] Y. Bar-Shalom and T. E. Fortmann. Tracking and Data Association. Academic Press, San Diego, CA, 1988.

[3] S. S. Blackman. Multiple-Target Tracking with Radar Applications. Artech House, Boston, MA, 1986.

[4] H. A. P. Blom and E. A. Bloem. Probabilistic data association avoiding track coalescence. IEEE Trans. Automat. Control, 45(2):247-259, 2000.

[5] H. A. P. Blom and E. A. Bloem. Joint particle filtering of multiple maneuvering targets from unassociated measurements. Journal of Advancement Information Fusion, 1:15-36, 2006.

[6] A. Doucet, N. de Freitas, and N. Gordon. Sequential Monte-Carlo Methods in Practice. Springer-Verlag, April 2001.

[7] N. J. Gordon, D. J. Salmond, and A. F. M. Smith. Novel approach to nonlinear/non-Gaussian Bayesian state estimation. IEE Proceedings $F$ Radar and Signal Processing, 140(2):107-113, 1993.

[8] C. Hue, J-P. Le Cadre, and P. Prez. Tracking multiple objects with particle filtering. IEEE Trans. Aerospace and Electronic Systems, 38(3):791-812, July 2002.

[9] A. H. Jazwinski. Stochastic processes and filtering theory. Academic Press, New York, 1970.

[10] T. Kirubarajan and Y. Bar-Shalom. Probabilistic data association techniques for target tracking in clutter. Proceedings of The IEEE, 92(3):536-557, 2004

[11] B. Kragel, S. Herman, and N. Roseveare. A comparison of methods for estimating track-to-track assignment probabilities. IEEE Trans. Aerospace and Electronic Systems, 2011. In Press.

[12] I. Kyriakides, D. Morrell, and A. Papandreou-Suppappola. Sequential Monte Carlo methods for tracking multiple targets with deterministic and stochastic constraints. IEEE Trans. Signal Process., 56(3):937948, 2008.

[13] S. Oh, S. Russell, and S. Sastry. Markov chain Monte Carlo data association for multi-target tracking. IEEE Trans. Automat. Control, 54(3):481-497, 2009.

[14] D. B. Reid. An algorithm for tracking multiple targets. IEEE Transactions on Automatic Control, 24:843-854, 1979.

[15] B. Ristic, S. Arulampalam, and N. Gordon. Beyond the Kalman Filter: Particle Filters for Tracking Applications. Artech House, Boston, MA, 2004.

[16] T. Yang, P. G. Mehta, and S. P. Meyn. Feedback particle filter with mean-field coupling. In Proc. of IEEE Conference on Decision and Control, pages 7909-7016, December 2011.

[17] T. Yang, P. G. Mehta, and S. P. Meyn. A mean-field controloriented approach to particle filtering. In Proc. of American Control Conference, pages 2037-2043, June 2011. 